\title{
Mechanism of metal activation of human hematopoietic prostaglandin D synthase
}

Tsuyoshi Inoue, Daisuke Irikura, Nobuo Okazaki, Shigehiro Kinugasa, Hiroyoshi Matsumura, Nobuko Uodome, Masaki Yamamoto, Takashi Kumasaka, Masashi Miyano, Yasushi Kai and Yoshihiro Urade

Nat. Struct. Biol. 10, 291-296 (2003).

The $y$-axes of Fig. 3a,b, $d$ in this manuscript were labeled incorrectly. The correct unit for the PDGS activity in these panels should be

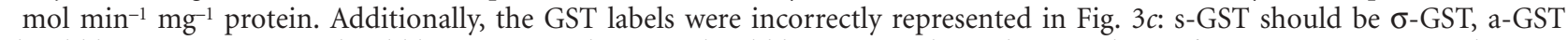
should be $\alpha$-GST; m-GST should be $\mu$-GST; and p-GST should be $\pi$-GST. The authors apologize for any inconvenience this may have caused. 